Monatsschrift f. Geburtshülfe u. Gynäkologie 1923;62:I-IV

\title{
Contents, Vol. 62, 1923
}

\section{Inhaltsverzeichnis.}

Seit»

Borell, H., Über die Bedeutung der Gabaston-Methode für

die Lösung der Plazenta 295

Bowing, K., Klinische und anatomische Betrachtungen

über ausgetragene Ovarialgravidität 127

Fink, K., Theoretisches und Praktisches vom Steißteratom 307 Fleischer, R., Ein Fall von

lymphzystisch degeneriertem

Ovar. 45

,-- , Selbstwendung oder Selbstentwicklung 50

Floris, B., Das Schicksal der Hydramnionkinder ... 55

Graff, E., Die Durchblasung nach Rubin zur Feststellung

der Wegsamkeit der Tuben. (Zu der Veröffentlichung

von H. Guthmann über den gleichen Gegenstand auf

Seite 10 des LIX. Bandes dieser Monatsschrift) . . 159

Grzechowiak, F., Die medikamentöse Beeinflussung des

Kapillarkreislaufes am Fingernagelfalz

Heil, K·, Über die Komplikation von Schwangerschaft

und Mastdarmkrebs 179

Hermstein, K., Die Resultate der hohen Naegele- und

Kielland-Zange . . 139

Herzfeld, B., Die Wehenschwäche und ihre Therapie . 201 Hinselmann, Ausgewählte Fragen der Eklampsieforschung.

(Fortsetzung aus Bd,.LIX, Heft 1/2).37

Hochenbichler, A., Über die prophylaktische Behand-

lung der Eklampsie mit künstlicher Höhensonne . . 269

Hofmeier, M., Über die Superfetatio beim Menschen . 1

Klee, F., und E. Hofmann, Über die Verwendbarkeit

des Retroplazentar- und Nabelvenenblutes zur Syphilis-

diagnose

21

K ü s t n e r, H., Der renale Diabetes während der Schwangerschaft in seiner Abhängigkeit von den Funktionen der

Drüsen mit innerer Sekretion 119

Martin, A., Der Kampf gegen die Krebserkrankung . . 241 Miltner, Th. v., Lobelin bei Asphyxie der Neugeborenen 60 Naujoks, H., Über intrauterine Skelettierung. .... 285 Neuwirth, K., Isoliprte Tuberkulose der Portio .... 163 Offermann, W., Über die Schwangerschaftsprophylaxe der puerperalen Mastitis 281

Santner, A., Wie beeinflußt die Zange die Kindersterblich-

keit unter der Geburt 289 
Scholten, J., und F. Voltz, Unsere Milzreizbestrahlungen

bei Menorrhagien und Metrorrhagièn 194

Sellheim, H., Fortschreitende Entwertung der Warnekros-

schen Dokumente 247

$\mathrm{XV}$ Inhaltsverzeichnis.

Seit $\beta$

S i e g e 1, P. W·, 1st der prophylaktische Kaiserschnitt beini

engen Becken berechtigt? $\quad .147$

Stübler, E., Primäres Tubenkarzinom und Tubentuberkulose 173

Vogt, E. , Neue Tatsachen und Probleme zur Lehre von

der Spermaimmunität 317

Voltz, F., Statistische Untersuchungen an 2000 Karzinom-

fällen 187

Enzinger, E., Die Berechtigung zu den kindstòtenden Operationen intra partum, von

verschiedenen Stand-punkten aus unter besonderer Berücksichtigung der moraltheologischen

Anschauungen dargestellt .... 69

Ellerbroek, N., Die Regelung des Berufs einer Wochen-

pflegerin 79

Frankl, 0., Über Endometrioma ovarii (Referat) ... 93

Heil, K., Eine literarische Anregung . 95

Sammelberichte.

Italienischer Literaturbericht. Von Prof. Dr. Fl. $\mathrm{c} \Gamma$ Erchia

in Bari-Genua 96

Die geburtshilfliche und gynäkologische Literatur in Finn-

land 1921, in schwedischer und finnischer Sprache. Von

Dr. Edw. A. Björkenheim in Helsingfors 321

Sammelbericht aus dem American Journal of Obstetrics and

Gynecology, St. Louis. Vol. I, Oktober 1920 bis Juni

1921. Von Dr. R Kuhn in München 325

Vereîns- und Literaturbeilage. Origînal-Sitzungsberichíe aus geburtshülflich-gynãkologäschen

Gesellschaften: Gynäkologische Gesellschaft in Breslau. Festsitzung zur

Feier des 20jährigen Bestehens der Gesellschaft am

4. November $1922 \ldots, \quad 109$

Sitzung vom 28. November $1922 \quad 214$

Mittelrheinische Gesellschaft für Geburtshülfe und Gynäko-

logie. 58. Sitzung vom 8. Juli lí 22217

Gesellschaft für Geburtshülfe und Gynäkologie zu Berlin.

Sitzung vom 27. Oktober $1922 \quad 226$

Nordostdeutsche Gesellschaft für Gynäkologie. 52. Sitzung

vom 25. November $1922 \quad .337$

Niederrheinisch-westfälische Gesellschaft für Gynäkologie

und Geburtshülfe. 69. Sitzung vom 18. November 1922343 Reîerate aus geburtshülflích-

gynäkologische $\pi$ Gesellschaften Ill, 231 Geburtshülflich-gynäkologische Mitteilungen aus anderen me-

dizînischen Gesellschaften und Vereinen ... 112, 232, 352

Buchbesprechungen 233, 353 
Literatur-Verzeíchnis 113, 234, 354

Peter Müller $\uparrow 211$

Personalnachríchten 108, 213, 336 